\section{Determinación y jerarquización de signos clínicos para diagnóstico temprano de xantomatosis cerebrotendinosa}

JAVIER VEGA V. ${ }^{1, \mathrm{a}}$, PAULINA SOLERVICENS R. ${ }^{1, \mathrm{~b}}$, ALBERTO MAIZ G. ${ }^{1}$, YUDITH PREISS C. ${ }^{2}$, PATRICIO MELLADO T. ${ }^{3}$, JOSÉ L. SANTOS M. ${ }^{1, \mathrm{c}, \mathrm{de}}$

\section{Determination and hierarchy of clinical signs for early detection of cerebrotendinous xanthomatosis}

Background: Cerebrotendinous Xanthomatosis (CTX) is an autosomal recessive disease caused by mutations in the CYP27A1 gene resulting in a decreased synthesis of bile acids. An early diagnosis and treatment would reduce the longterm complications observed in this disease. Aim: To identify and hierarchize initial clinical signs of CTX to establish an early diagnostic suspicion index. Material and Methods: Clinical information was collected from 387 patients diagnosed with CTX, published in MEDLINE between 1968 and 2016. Clinical manifestations were identified, determining their prevalence and age of onset. Sensitivity, specificity and the positive Likelihood ratio $(L R+)$ was calculated for each clinical sign evaluated. Results: The average ages for early symptoms' onset and CTX diagnosis were $13.3 \pm 10.6$ years and $34.6 \pm 12.6$ years respectively. The early clinical signs and their respective $L R+$ were: juvenile cataracts (143), epilepsy (81), chronic diarrhea (15.6) and psychomotor development delay (3.4). The presence of consanguinity among parents resulted in a $L R+$ of 31 . The combination of two early signs increased the post-test probability to $30 \%$. If the early diagnostic criteria would have been applied in three Chilean patients with diagnosis of CTX, their disease would have been diagnosed from 12 to 25 years earlier. Conclusions: The use of a hierarchical system of predictive clinical signs allows an early screening of CTX, which may avoid the natural progression of the disease using an appropriate treatment.

(Rev Med Chile 2018; 146: 745-752)

Key words: Bile Acids and Salts; Genetics; Xanthomatosis, Cerebrotendinous.

\author{
'Departamento de Nutrición, \\ Diabetes y Metabolismo. \\ Escuela de Medicina. Pontificia \\ Universidad Católica de Chile. \\ Santiago, Chile. \\ ${ }^{2}$ Hospital DIPRECA. Santiago, \\ Chile. \\ ${ }^{3}$ Departamento de Neurología. \\ Escuela de Medicina. Pontificia \\ Universidad Católica de Chile. \\ Santiago, Chile. \\ aResidente de Nutrición Clínica y \\ Diabetología. \\ ${ }^{\text {b}}$ Tesista. Programa de Magister \\ en Nutrición. Pontificia \\ Universidad Católica de Chile. \\ 'Bioquímico. \\ ${ }^{\mathrm{d} P h D}$. \\ eMSc. \\ Trabajo finaciado por fondos \\ propios del Departamento \\ de Nutrición, Diabetes y \\ Metabolismo de la Facultad \\ de Medicina de la Pontificia \\ Universidad Católica de Chile. \\ Los autores declaran no tener \\ conflictos de interés.
}

Recibido el 1 de septiembre de 2017, aceptado el 28 de mayo de 2018.

Correspondencia a: José Luis Santos M. Departamento de Nutrición, Diabetes y Metabolismo. Escuela de Medicina. Edificio de Gastroenterología, 4 Piso. Pontificia Universidad Católica de Chile. Avenida Libertador Bernardo O `Higgins 340. Santiago, Chile. Teléfonos: (56 2) 3543862, (56 2) 3543865 ,

(56 2) 3543868

jsantos@med.puc.cl

\section{L} a xantomatosis cerebrotendinosa (XCT) es una enfermedad genética rara del metabolismo de los ácidos biliares, de carácter autosómico recesivo, producida por mutaciones en el gen CYP27A1, que codifica para la enzima mitocondrial 27 -esterol hidroxilasa ${ }^{1,2}$. Se han descrito más de 50 mutaciones en este gen, de las cuales, la mitad se localiza entre los exones 6 y 8 y $45 \%$ corresponde a mutaciones que generan cambios de aminoácidos en la cadena peptídica ${ }^{3}$. Estas alteraciones genéticas producen una disminución en la síntesis de ácidos biliares primarios a partir de colesterol, como los ácidos quenodeoxicólico (AQDC) y cólico (AC) y un exceso de los meta- 
bolitos derivados de esta vía, principalmente la 7 $\alpha$-hidroxi-colesten-3-ona (también llamado C4) y su producto de hidroxilación, el colestanol. El depósito de este último en diferentes tejidos, en particular tendones y cerebro, genera las manifestaciones clínicas típicamente descritas ${ }^{4}$, sin que exista una relación clara entre el genotipo identificado y el fenotipo del paciente, pudiendo encontrarse distintas presentaciones de la enfermedad para una misma mutación ${ }^{5}$. La administración exógena de AQDC actúa suprimiendo la síntesis de ácidos biliares, ejerciendo un efecto de retroalimentación negativa y normalizando los niveles de colestanol en plasma.

Mediante dos vías metabólicas complementarias (Figura 1), la síntesis de ácidos biliares en el hígado representa el mecanismo de excreción de colesterol ${ }^{6,7}$. La vía clásica o "neutra" se inicia en el retículo endoplasmático con la hidroxilación del colesterol en la posición 7, por acción de la enzima colesterol $7 \alpha$-hidroxilasa (CYP7A1). Posteriormente, la 27-esterol hidroxilasa cataliza la oxidación de la cadena lateral de colesterol para la síntesis de los ácidos cólico y quenodeoxicólico, los cuales representan aproximadamente el 31 y $45 \%$ de todos los ácidos biliares, respectivamente. En la vía alternativa o "acídica", la 27-esterol hidroxilasa ubicada en la membrana interna de la mitocondria cataliza el primer paso, oxidando el colesterol a 27-hidroxicolesterol, para finalmente producir $\mathrm{AQDC}^{6}$. Se ha descrito que tanto los ácidos quenodeoxicólico y cólico pueden regular la expresión de genes implicados en su síntesis. Por lo tanto, una disminución de AQDC por una deficiencia de la CYP27A1 conduce a una retroalimentación positiva hacia la vía metabólica mediada por la enzima $7 \alpha$-hidroxilasa. Esta sobrestimulación eleva los niveles de $7 \alpha$-hidroxi-4-colesten-3-ona (C4), un precursor del colestanol ${ }^{1,2,8}$.

La prevalencia de la XCT varía según población y etnia, pero se estima que es menor a 5 casos por 100.000 habitantes en la mayoría de los países. Considerando solamente la mutación p.R395C, que es la más frecuente tanto en la población española como chilena ${ }^{9,10}$, la frecuencia de XCT en población de origen europeo se puede estimar cercana a $1 / 50.000^{11}$. Al tratarse de una enfermedad con herencia autosómica recesiva, algunos de los casos descritos están causados por mutaciones en estado de heterocigoto compuesto, mientras

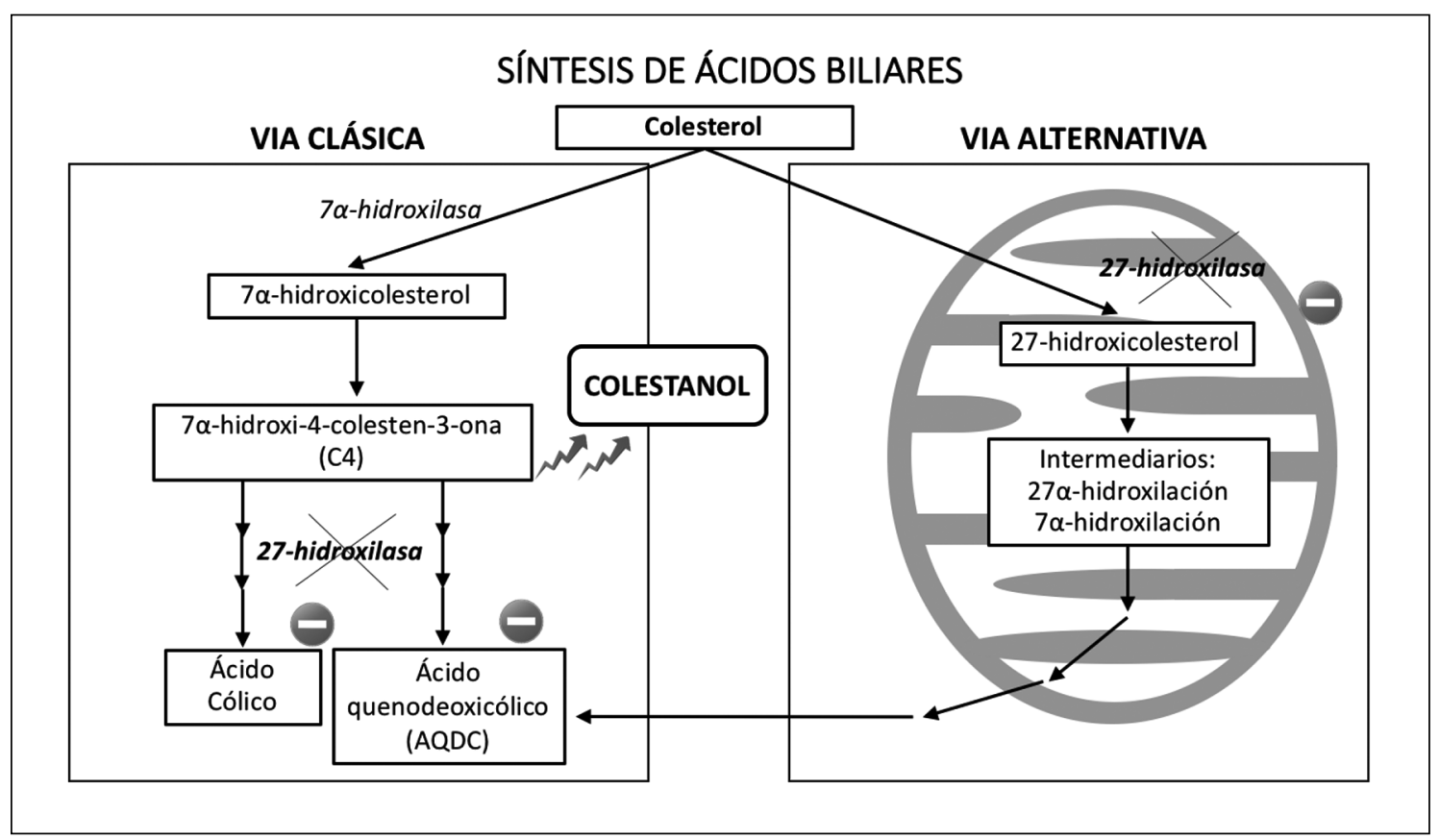

Figura 1. Vías metabólicas de la síntesis de ácidos biliares. El déficit de la 27-hidroxilasa aumenta los niveles plasmáticos de colestanol y C4. 
que otros derivan de enlaces consanguíneos. En cualquier caso, el diagnóstico de XCT está aparentemente subestimado. Sobre la base del estudio de una cohorte con secuenciación de 60.000 exomas de adultos de diferentes grupos étnicos, se han encontrado numerosas variantes de CYP27A1 con efecto patogénico, existiendo una mutación (p.P384L) con una frecuencia alélica superior a $1 \%$ en poblaciones latinas, europeas y asiáticas. De acuerdo con este estudio, la incidencia de la enfermedad se situaría en rangos de 1:36.072 a 1:468.624, según la población y grupo étnico analizados $^{12}$. En Chile han sido publicados 5 casos de pacientes con XCT, con diagnóstico clínico y genético completo ${ }^{10} \mathrm{y}$ (Preiss Y. Características clínicas y genéticas de cinco pacientes chilenos con diagnóstico clínico y serológico de Xantomatosis Xerebrotendinosa". Tesis para optar al grado de Magister en Nutrición. Pontificia Universidad Católica de Chile. 2012).

El diagnóstico de la xantomatosis cerebrotendinosa se basa en la sospecha clínica ante la presencia de 2 de los 4 signos descritos como cardinales ${ }^{13}$ y que se presentan en forma progresiva en el tiempo: 1) diarrea crónica en la infancia; 2) cataratas juveniles; 3 ) xantomas tendíneos y 4) síntomas neurológicos. La diarrea crónica y de difícil manejo se describe por sobre el 50\% de los casos durante la etapa de lactante y sería producido por la disminución de ácidos biliares y su reemplazo por alcoholes biliares desde las vías metabólicas involucradas ${ }^{2,9}$. Las cataratas aparecen en la primera década de la vida y afectan a $75 \%$ de los pacientes ${ }^{14}$. También se describe en menor frecuencia esclerosis de vasos retinianos y depósitos similares a colesterol ${ }^{2,13}$. Los xantomas tendíneos se originan desde la segunda década de la vida y están conformados por tejido conectivo asociado a depósitos de colestanol y colesterol, afectando principalmente articulaciones extensoras como el tendón de Aquiles, codo, mano y nivel cervical ${ }^{1}$. Se han descrito una gran variedad de síntomas neurológicos relacionados a la XCT, sin embargo, la epilepsia y el parkinsonismo son habituales al comienzo de la enfermedad ${ }^{2}$. Mediante neuroimágenes se puede observar atrofia cerebelosa, alteraciones de la señal de la sustancia blanca e hiperintensidades simétricas en los núcleos dentados ${ }^{15}$. Con menor frecuencia se describe osteoporosis prematura, ateroesclerosis precoz, colestasia neonatal y cardiopatía coronaria ${ }^{13}$, aun cuando el nivel de colesterol plasmático está normal o incluso bajo.

Aunque el promedio de inicio de los síntomas es cercano a los 19 años de edad, el diagnóstico se establece frecuentemente en la cuarta década de la vida, representando un retraso aproximado del diagnóstico de más de 15 años $^{2}$. Frente a la presencia de síntomas sugerentes, la confirmación diagnóstica se realiza con la constatación de niveles elevados de colestanol plasmático, C4 plasmático $\mathrm{o}$ análisis genético ${ }^{5}$. El tratamiento en etapas precoces evitaría la progresión de la enfermedad y sus complicaciones neurológicas, cognitivas y osteotendíneas $^{16}$. Dado lo anterior, se deriva que la determinación de aquellos signos clínicos precoces relevantes para identificar en forma temprana esta patología permitiría el tratamiento oportuno de los pacientes. Por tanto, los objetivos de este trabajo son determinar y jerarquizar los primeros signos de aparición de xantomatosis cerebrotendinosa para establecer una sospecha diagnóstica precoz que permita un tratamiento apropiado de la enfermedad.

\section{Sujetos y Métodos}

\section{Pacientes}

Se realizó una búsqueda de casos clínicos con diagnóstico de XCT publicados en la literatura, a partir de la base de datos MEDLINE entre los años 1968 y 2016, utilizando los términos: "Xanthomatosis, Cerebrotendinous" [Mesh], "Cerebrotendinous xantomatosis", "brain xanthoma", "tendon xanthoma", "cholestanol" y "CYP27A1". Se incluyeron dentro del análisis aquellos artículos que mencionaran la edad de aparición de los signos clínicos y de laboratorio observados. El diagnóstico se estableció según las características clínicas de acuerdo a los criterios descritos previamente, con confirmación bioquímica de niveles plasmáticos de colestanol y, en algunos casos, por confirmación genética de la mutación.

\section{Recolección y evaluación de datos}

A partir de la información obtenida, se revisaron los antecedentes clínicos, epidemiológicos y de consanguinidad de los pacientes y se registró la edad de inicio de los siguientes síntomas y signos: xantomas tendíneos, diarrea crónica originada en la infancia, epilepsia, cataratas juveniles, signos 
piramidales, signos extrapiramidales, discapacidad intelectual, alteraciones psiquiátricas, parkinsonismo, polineuropatía, lesiones en el núcleo dentado en imágenes, signos cerebelares, enfermedad ateroesclerótica y osteoporosis. También se recopilaron la edad del paciente al momento del diagnóstico, el antecedente de consanguinidad de los padres y la presencia de colestanol elevado. Se utilizó la prevalencia descrita para cada signo en la población general ${ }^{17-20}$ como estimación de la probabilidad pretest de los signos clínicos analizados.

\section{Análisis estadístico}

Para cada signo o síntoma se calculó su sensibilidad (S), especificidad (E) y edad de aparición. Se utilizaron datos de prevalencia de las comorbilidades presente en población general infantil para el cálculo de especificidad, ya que en este estudio solo se recopiló información de pacientes afectados con $\mathrm{XCT}$ y no de controles sanos. De los valores de S y E, se obtuvo el Positive Likelihood Ratio (LR+), que representa la razón entre la probabilidad de tener determinado resultado del test en la población con la condición versus tener el mismo resultado en la población sin la condición ${ }^{21}$. Se aplicó en forma retrospectiva la jerarquización de los signos clínicos de sospecha diagnóstica en 3 pacientes chilenos que presentaban la enfermedad y cuyos casos clínicos han sido reportados ${ }^{10,22}$.

\section{Resultados}

Un total de 628 artículos sobre xantomatosis cerebrotendinosa fueron encontrados a través de la base de datos MEDLINE, de los cuales se seleccionaron 151 por contar con reportes de casos clínicos. Se recopilaron 387 pacientes con diagnóstico confirmado de XCT. De estos, 142 eran mujeres, $72,2 \%$ tenía antecedente de algún familiar de primer grado con el mismo diagnóstico y en $30,8 \%$ de los casos existía consanguinidad entre los padres. La prevalencia de los signos clínicos analizados se encuentra en la Figura 2A. En la Figura 2B se detalla el promedio y el rango de edad de aparición. La edad media, la desviación estándar y la mediana del inicio de los síntomas evaluados fue de 13,3 \pm 10,6 años y 10 años (rango entre 0 y 50 años). En cambio, la edad media, desviación estándar y mediana del diagnóstico fue $34,6 \pm 12,6$ años y 35 años, respectivamente (rango entre 0 y 57 años). Los signos clínicos de aparición más precoces que se encontraron, su promedio, desviación estándar y mediana fueron: diarrea crónica (11,3 \pm 14 y 5 años), discapacidad intelectual (12,8 $\pm 11,8$ y 8 años), epilepsia (13,5 $\pm 13,3$ y 9 años) y cataratas $(18,2 \pm 11,2$ y 17 años). La totalidad de los casos descritos presentaron niveles de colestanol plasmáticos elevados. La Tabla 1 muestra la sensibilidad, especificidad y LR+ para cada signo precoz, calculados a partir de la prevalencia de esa patología en la población infantil.

Al aplicar el LR+ para cada signo precoz a un paciente con sospecha de xantomatosis cerebrotendinosa, considerando una prevalencia de enfermedad de 0,00005 , la probabilidad postest frente a la presencia de diarrea fue de $0,078 \%$, para retraso del desarrollo psicomotor $0,018 \%$, epilepsia $0,41 \%$, cataratas $0,71 \%$ y para el antecedente de consanguinidad $0,22 \%$. Sin embargo, si se combinan dos de esos antecedentes (Tabla 2 ), los signos que obtienen mayor probabilidad

Tabla 1. Ponderación de signos clínicos precoces en sospecha de xantomatosis cerebrotendinosa

\begin{tabular}{|lccc|}
\hline Signo & Sensibilidad & Especificidad & Likelihood Ratio Positivo \\
\hline Diarrea crónica & 0,62 & 0,96 & 15,6 \\
Retraso desarrollo psicomotor & 0,39 & 0,89 & 3,6 \\
Epilepsia & 0,49 & 0,99 & 81,9 \\
Cataratas juveniles & 0,86 & 0,99 & 143,8 \\
\hline Consanguinidad & 0,31 & 0,99 & 44,6 \\
\hline
\end{tabular}

La tabla muestra la sensibilidad, especificidad y Likelihood Ratio positivo para los 4 signos clínicos identificados como precoces para la progresión a XCT, además del antecedente de consanguinidad y la presencia de 1 o más signos precoces, independiente de cuáles estos sean. 


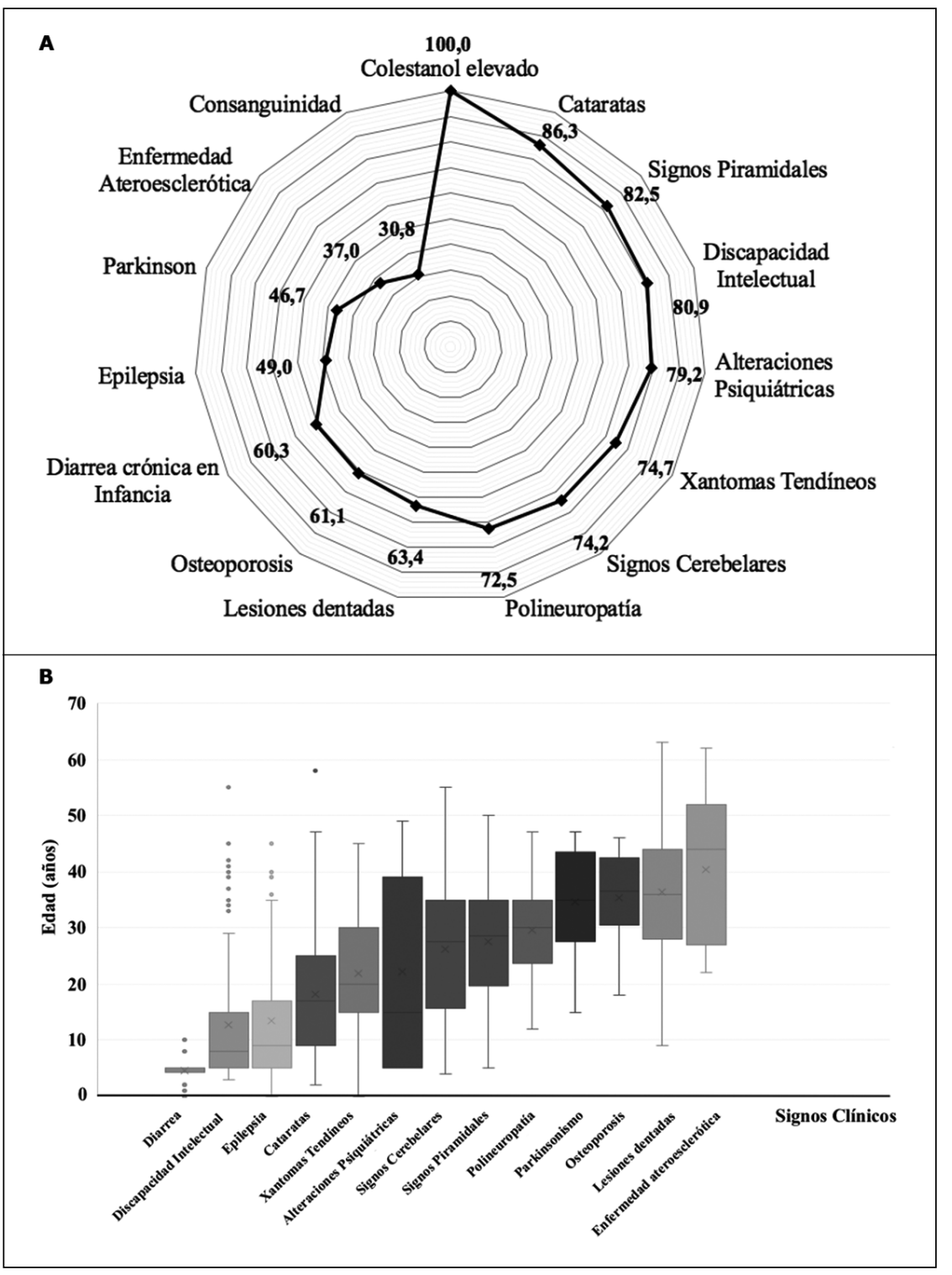

Figura 2. A. Prevalencia de los síntomas y signos clínicos en xantomatosis cerebrotendinosa. Los valores se encuentran expresados en porcentaje. B. Rangos de edad de aparición, media, mediana y desviación estándar de los signos analizados.

Tabla 2. Probabilidad postest de presentar xantomatosis cerebrotendinosa al combinar la detección de 2 signos precoces (expresada en porcentajes)

\begin{tabular}{|lccccc|}
\hline Signo & Diarrea & RDSM & Epilepsia & Cataratas & Consanguinidad \\
\hline Probabilidad basal & 0,077 & 0,017 & 0,41 & 0,71 & 0,22 \\
\hline Diarrea & & 0,28 & 5,98 & 10,02 & 3,35 \\
RDSM & & 1,45 & 2,51 & 0,79 \\
Epilepsia & & & 36,89 & 15,41 \\
Cataratas & & & & 24,24 \\
\hline
\end{tabular}

RDSM: Retraso del Desarrollo Psicomotor. 
postest de presentar XCT fueron la combinación de cataratas y epilepsia, y cataratas y consanguinidad, siendo en ambos casos una probabilidad mayor a $24 \%$.

Adicionalmente, tres casos de pacientes chilenos descritos en la literatura se analizaron en forma retrospectiva. El inicio de sus síntomas fue antes de los 5 años, caracterizados por diarrea crónica, cataratas y alteraciones neurológicas. Sin embargo, el diagnóstico más precoz no se logró determinar hasta los 17 años, mientras que en los otros pacientes se estableció sobre los 30 años de edad. Por tanto, el retraso en el diagnóstico en estos tres pacientes chilenos tuvo lugar en un rango entre 12 y 25 años.

\section{Discusión}

La xantomatosis cerebrotendinosa en una enfermedad genética con muy baja prevalencia y su diagnóstico se realiza frecuentemente en etapas avanzadas de la enfermedad, dificultando el tratamiento oportuno con AQDC. Este trabajo ha pretendido identificar aquellos signos clínicos de aparición más temprana en el curso de la enfermedad. Si se considera que la prevalencia de $\mathrm{XCT}$ es baja, la presencia de cualquiera de estos signos aumenta de forma notoria la probabilidad postest a un valor que, cuando se combinan dos signos precoces simultáneamente, la probabilidad postest aumenta hasta por sobre $30 \%$, como ocurre en la presencia en conjunto de cataratas y epilepsia. Si se identifican tres signos combinados, la probabilidad de presentar XCT se eleva sobre $80 \%$, sin embargo, esto ocurre solo en 7,5\% de los pacientes. En cambio, la prevalencia de dos o más signos precoces en pacientes con la enfermedad es mayor $(34,6 \%)$.

En este trabajo hemos evidenciado adicionalmente que el tiempo transcurrido entre la aparición de los primeros síntomas de la enfermedad y su diagnóstico es, aproximadamente, de 21 años en pacientes reportados en literatura mundial. En este sentido, se ha constatado que el tratamiento precoz permite una evolución favorable, incluso con la reversión de algunos de los síntomas clásicos. Berginer y cols. ${ }^{24}$ evaluaron a cuatro pacientes de una misma familia con XCT, encontrando que los dos miembros más jóvenes (edad de 2 y 7 años) al momento del diagnóstico estaban asintomáti- cos, a diferencia de los miembros mayores de la familia (de 16 y 27 años de edad), quienes ya presentaban la totalidad de los síntomas. Al cabo de 14 años de tratamiento con AQDC, los pacientes jóvenes persistieron asintomáticos e incluso con reversión total de las manifestaciones bioquímicas e imageneológicas, en cambio, los pacientes con inicio de tratamiento más tardío, si bien manifestaron una leve mejoría clínica, continuaron con las complicaciones de la evolución natural de la enfermedad. Adicionalmente, se han reportado resultados controversiales sobre la efectividad del tratamiento en etapas avanzadas de la enfermedad, dado que el deterioro neurológico no cambia o sigue su curso natural'.

Actualmente, el número de pacientes descritos en población chilena con diagnóstico clínico y de laboratorio es de 5 casos. Sin embargo, según el cálculo obtenido a partir de la cohorte con secuenciación de exomas ${ }^{12}$, los pacientes con XCT a nivel nacional podrían llegar a ser más de 40 , reflejando un subdiagnóstico a nivel local. Por lo anterior, una mayor sospecha diagnóstica y una confirmación precoz, permitiría acceder al tratamiento en etapas iniciales.

El diagnóstico molecular de XCT se realiza mediante test genético o, más frecuentemente, vía medición plasmática de niveles de colestanol o 7- $\alpha$-hidroxi-4-colesten-3-ona (C4). Con el fin de simplificar el diagnóstico y lograr un tamizaje masivo de XCT, se han evaluado pruebas en orina que identifican el colestanpentol, un alcohol biliar que se encuentra elevado en estos pacientes ${ }^{24}$. La aplicación de alguno de estos métodos permitiría plantear la posibilidad de incluir la XCT en la pesquisa neonatal de enfermedades relacionadas con errores innatos del metabolismo, incrementando así su diagnóstico.

Los pilares de manejo del paciente con XCT incluyen el tratamiento farmacológico con ácido quenodeoxicólico (AQDC) y el tratamiento sintomático de las complicaciones asociadas. La administración exógena de AQDC inhibe la producción de ácido biliar, por una disminución de la producción del metabolito intermedio, C4, reduciendo así los niveles de colestanol en plasma y la eliminación de alcoholes biliares. La administración conjunta de estatinas parece potenciar este efecto ${ }^{25}$.

Una limitación de este estudio es que los cálculos fueron realizados a partir de la prevalencia 
obtenida en la literatura, y para el caso de xantomatosis cerebrotendinosa, la prevalencia descrita tiene una alta variación dependiendo de la población estudiada. La misma situación ocurre con los signos y síntomas precoces analizados. Por este motivo, para el cálculo de E y LR+ hemos utilizado prevalencias que se asemejaban a la población española o chilena, considerando la semejanza genética entre ambas poblaciones. Otro factor a considerar es el posible sesgo de selección por idioma en la búsqueda de casos clínicos.

En conclusión, en este trabajo hemos logrado identificar los signos clínicos que aparecen en las primeras etapas de la xantomatosis cerebrotendinosa, como son la presencia de cataratas juveniles, epilepsia y diarrea crónica en la infancia. Utilizando un índice de sospecha clínica basado en estos signos, podemos adelantar significativamente el diagnóstico. Este trabajo muestra que existe un subdiagnóstico de esta enfermedad, así como evidencia la importancia de realizar una identificación temprana y un tratamiento precoz con ácido quenodeoxicólico, lo que evitaría la progresión natural de la enfermedad.

\section{Referencias}

1. Preiss Y, Santos JL, Smalley SV, Maiz A. Xantomatosis cerebrotendinosa: aspectos fisiopatológicos, clínicos y genéticos. Rev Med Chile 2014; 142 (5): 616-22.

2. Nie S, Chen G, Cao X, Zhang Y. Cerebrotendinous xanthomatosis: a comprehensive review of pathogenesis, clinical manifestations, diagnosis, and management. Orphanet J Rare Dis 2014; 9: 179.

3. Lorbek G, Lewinska M, Rozman D. Cytochrome P450s in the synthesis of cholesterol and bile acids--from mouse models to human diseases. FEBS J 2012; 279 (9): 1516-33.

4. Båvner A, Shafaati M, Hansson M, Olin M, Shpitzen $\mathrm{S}$, Meiner V, et al. On the mechanism of accumulation of cholestanol in the brain of mice with a disruption of sterol 27-hydroxylase. J Lipid Res 2010; 51 (9): 2722-30.

5. Gallus GN, Dotti MT, Federico A. Clinical and molecular diagnosis of cerebrotendinous xanthomatosis with a review of the mutations in the CYP27A1 gene. Neurol Sci 2006; 27 (2): 143-9.

6. Hylemon PB, Zhou H, Pandak WM, Ren S, Gil G, Dent P. Bile acids as regulatory molecules. J Lipid Res 2009; 50 (8): 1509-20.

7. Norlin M, Wikvall K. Enzymes in the conversion of cholesterol into bile acids. Curr Mol Med 2007; 7 (2): 199-218.

8. Björkhem I, Boberg KM. Inborn errors in bile and biosynthesis and storage of sterols other than cholesterol. En: Scriver CR, Beaudet AL, Sly WS, Valle D, Editores, The Metabolic and Molecular Basis of Inherited Disease, 7th ed. McGraw- Hill, New York; 1995. p 2073 99.

9. Pilo-de-la-Fuente B, Jiménez-Escrig A, Lorenzo JR, Pardo J, Arias M, Ares-Luque A, et al. Cerebrotendinous xanthomatosis in Spain: clinical, prognostic, and genetic survey. Eur J Neurol 2011; 18 (10): 1203-11.

10. Smalley SV, Preiss Y, Suazo J, Vega JA, Angellotti I, Lagos CF, et al. Novel splice-affecting variants in CYP27A1 gene in two Chilean patients with Cerebrotendinous Xanthomatosis. Genet Mol Biol 2015; 38 (1): 30-6.

11. Lorincz MT, Rainier S, Thomas D, Fink JK. Cerebrotendinous xanthomatosis: possible higher prevalence than previously recognized. Arch Neurol 2005; 62 (9): 1459-63.

12. Appadurai V, De Barber A, Chiang PW, Patel SB, Steiner RD, Tyler C, et al. Apparent underdiagnosis of Cerebrotendinous Xanthomatosis revealed by analysis of 60,000 human exomes. Mol Genet Metab 2015; 116 (4): 298-304.

13. Degos B, Nadjar Y, Amador M del M, Lamari F, Sedel F, Roze E, et al. Natural history of cerebrotendinous xanthomatosis: a paediatric disease diagnosed in adulthood. Orphanet J Rare Dis 2016; 11: 41.

14. Moghadasian MH. Cerebrotendinous xanthomatosis: clinical course, genotypes and metabolic backgrounds. Clin Invest Med 2004; 27 (1): 42-50.

15. Federico A, Dotti MT. Cerebrotendinous xanthomatosis. Neurology 2001; 57 (9): 1743.

16. Yahalom G, Tsabari R, Molshatzki N, Ephraty L, Cohen $\mathrm{H}$, Hassin-Baer S. Neurological outcome in cerebrotendinous xanthomatosis treated with chenodeoxycholic acid: early versus late diagnosis. Clin Neuropharmacol 2013; 36 (3): 78-83.

17. Fine KD, Schiller LR. AGA technical review on the evaluation and management of chronic diarrhea. Gastroenterology 1999; 116 (6): 1464-86.

18. Russ SA, Larson K, Halfon N. A national profile of childhood epilepsy and seizure disorder. Pediatrics 2012; 129 (2): 256-64.

19. Sheeladevi S, Lawrenson JG, Fielder AR, Suttle CM. Global prevalence of childhood cataract: a systematic review. Eye (Lond) 2016; 30 (9): 1160-9.

20. Schonhaut L, Álvarez J, Salinas P. El pediatra y la evaluación del desarrollo psicomotor. Rev Chil Pediatr 2008; 79 (Supl. 1): 26-31. 
21. Salech F, Mery V, Larrondo F, Rada G. Estudios que evalúan un test diagnóstico: interpretando sus resultados. Rev Med Chile 2008; 136 (9): 1203-8.

22. Filippi J, Irarrázaval S, Peredo P, Mellado P. Xantomatosis cerebrotendínea: Una causa de aumento de volumen bilateral del tendón de Aquiles. Caso clínico. Rev Med Chile 2009; 137: 815-20.

23. Berginer VM, Gross B, Morad K, Kfir N, Morkos S, Aaref $\mathrm{S}$, et al. Chronic diarrhea and juvenile cataracts: think cerebrotendinous xanthomatosis and treat. Pedia- trics 2009; 123 (1): 143-7.

24. Pitt JJ. High-throughput urine screening for SmithLemli-Opitz syndrome and cerebrotendinous xanthomatosis using negative electrospray tandem mass spectrometry. Clin Chim Acta 2007; 380 (1-2): 81-8.

25. Luyckx E, Eyskens F, Simons A, Beckx K, Van West D, Dhar M. Long-term follow-up on the effect of combined therapy of bile acids and statins in the treatment of cerebrotendinous xanthomatosis: a case report. Clin Neurol Neurosurg 2014; 118: 9-11. 